\title{
Robotic Tentacles with Three-Dimensional Mobility Based on Flexible Elastomers
}

\section{Citation}

Martinez, Ramses V., Jamie L. Branch, Carina R. Fish, Lihua Jin, Robert F. Shepherd, Rui M. D. Nunes, Zhigang Suo, and George M. Whitesides. 2013. "Robotic Tentacles with ThreeDimensional Mobility Based on Flexible Elastomers." Advanced Materials 25 (2) (January 11): 205-212.

\section{Published Version}

doi:10.1002/adma.201203002

\section{Permanent link}

http://nrs.harvard.edu/urn-3:HUL.InstRepos:12388816

\section{Terms of Use}

This article was downloaded from Harvard University's DASH repository, and is made available under the terms and conditions applicable to Open Access Policy Articles, as set forth at http:// nrs.harvard.edu/urn-3:HUL.InstRepos:dash.current.terms-of-use\#OAP

\section{Share Your Story}

The Harvard community has made this article openly available.

Please share how this access benefits you. Submit a story.

\section{Accessibility}


Robotic Tentacles with Three-Dimensional Mobility Based on Flexible Elastomers

By Ramses V. Martinez ${ }^{1}$, Jamie L. Branch ${ }^{1}$, Carina R. Fish ${ }^{1}$, Lihua Jin ${ }^{4}$, Robert F. Shepherd ${ }^{1}$, Rui M. D. Nunes ${ }^{1}$, Zhigang Suo ${ }^{2,4}$, and George M. Whitesides ${ }^{1,2,3^{*}}$

[*] Prof. G. M. Whitesides

${ }^{1}$ Department of Chemistry and Chemical Biology, Harvard University, 12 Oxford Street, Cambridge, MA 02138 (USA).

${ }^{2}$ Kavli Institute for Bionano Science \& Technology, Harvard University, 29 Oxford Street, Cambridge, MA 02138 (USA).

${ }^{3}$ Wyss Institute for Biologically Inspired Engineering, Harvard University, 60 Oxford Street, Cambridge, MA 02138 (USA).

E-mail: gwhitesides@gmwgroup.harvard.edu

Dr. R. V. Martinez, J. L. Branch, C.R. Fish, R. F. Shepherd, R. M. D. Nunes

${ }^{1}$ Department of Chemistry and Chemical Biology, Harvard University, 12 Oxford Street, Cambridge, MA 02138 (USA).

L. Jin

${ }^{4}$ School of Engineering and Applied Sciences, Harvard University, 29 Oxford Street, Cambridge, MA 02138 (USA).

Prof. Z. Suo

${ }^{4}$ School of Engineering and Applied Sciences, Harvard University, 29 Oxford Street, Cambridge, MA 02138 (USA).

${ }^{2}$ Kavli Institute for Bionano Science \& Technology, Harvard University, 29 Oxford Street, Cambridge, MA 02138 (USA).

Keywords: Soft Robotics, Tentacles, Pneumatic Actuators, Three-dimensional Motion, Composites.

The remarkable flexibility and dexterity of the tongues of mammals and lizards, the trunks of elephants, and other biological muscular systems ${ }^{[1]}$ inspire new designs for actuators and robots. ${ }^{[2]}$ The octopus arm, for example, is a non-rigid structure that has a very large number of degrees of freedom (DOFs), the ability to bend in all directions, high dexterity, and astonishing capability for fine manipulation. ${ }^{[3]}$ In robotics, researchers have developed a variety of trunk-like manipulators using rigid structures and electric motors with cable tendons for actuation. ${ }^{[4,5]}$ These hard robotic structures — structures based on multiple flexible 
joints connected by stiff links - are often heavy, and their control is complicated and expensive. Moreover, their underlying structures make it difficult to manipulate objects with parts of their arms other than their specialized end effectors.

"Soft" robots—robots composed of flexible components that provide multiple degrees of freedom - have many useful capabilities, including the abilities to deform their shape, to manipulate delicate objects, to conform to their surroundings, and to move in cluttered and/or unstructured environments. ${ }^{[6-8]}$ The flexibility of soft actuators offers potentially useful approaches to problems in robotics, and to the design of actuators, grippers, and other soft machines. They can also take advantage of often highly non-linear responses to actuation to accomplish, relatively simply, types of complex motions and tasks that are more difficult to accomplish using hard machines and conventional controllers. Soft pneumatic robots based on flexible elastomers, as one simple example, can distribute pressure uniformly over large areas without elaborate controls; this capability makes it possible for them to manipulate fragile and irregular objects. ${ }^{[6,9]}$

The mobility of the soft robotic structures with which we have worked-structures actuated by the expansion of elastomeric pneumatic networks - have been limited to a single bending mode in the direction defined by the anisotropy of the pneumatic expansion. ${ }^{[6,7]} \mathrm{We}$ wished to improve the motion capabilities of these systems, and specifically to fabricate entirely soft robotic actuators with three-dimensional (3D) motion, low cost, and simplicity of control.

This communication describes the design and fabrication of soft tentacles based on micropneumatic networks spatially distributed at the interface of two different elastomers; these composite elastomeric structures enable complex 3D motion of the tentacles. We demonstrate both the range of motions open to these tentacles, and their capability to grip and manipulate objects with complex shapes. We also extend the capabilities of these soft 
actuators by embedding functional components (for example, a needle for delivering fluid, a video camera, and a suction cup) in them. We further demonstrate that modifying the texture of the surface of the tentacles can improve their adhesion to slippery surfaces.

There are many demonstrations of hard robots that show highly flexible motion; these include multi-jointed trunk-like structures. ${ }^{[4,5]}$ By combining cable-tendon actuators with a bendable backbone made of alternating rigid and soft disks, Buckingham et al. ${ }^{[10]}$ built trunklike robots called "snake-arm robots" (OC Robotics, UK), which have been commercialized. It is, thus, possible to achieve some of the capabilities of soft structures even when the underlying actuating materials are hard. It is, however, difficult for hard robots to operate in certain types of unstructured and congested environments, because their underlying skeletons are rigid. ${ }^{[11]}$

Electroactive polymers (EAPs) have many characteristics that make them suitable for soft robots: low weight, fracture tolerance, rapid response, and relatively large actuation strain. ${ }^{[12,13]}$ There have been a number of approaches to arm-like structures based on EAPs, but none have been extensively developed or deployed. ${ }^{[14,15]}$ These soft actuators have the disadvantage of requiring high actuation voltages (up to $150 \mathrm{MV} / \mathrm{m}$ ); EAPs also degrade rapidly in use. ${ }^{[16]}$

Pneumatic artificial muscles (PAMs) - pneumatic actuators that consist of a thin, flexible, tubular membrane with fiber reinforcement—-have been used to make trunk-like soft robotic manipulators. ${ }^{[11,17,18]}$ For example, Pritts and Rahn (2004) developed a soft manipulator with two sections, each of which provide two axis bending. ${ }^{[19]}$ Half of the actuators in each section are extensors and the other half are contractors. "OctArm VI" is a soft robotic manipulator in which PAMs are connected together in groups of three and six to create three independently actuated sections that allow manipulation of objects. ${ }^{[17,18]}$ 
Our previous work in soft robotics has demonstrated the use of pneumatic networks (PneuNets) - fabricated in elastomeric materials using soft lithographic techniques highly developed in microfluidics — to serve as soft robotic actuators. ${ }^{[6,7]}$ Here we use these systems to generate new types of elastomeric soft actuators that allow 3D motion. This type of actuator is simple to fabricate, flexible in its operation, and inexpensive.

We used two silicone elastomers (Ecoflex 00-30, Smooth-on, http://www.smoothon.com) and a polydimethylsiloxane (PDMS; Dow Corning Sylgard 184) to fabricate soft tentacles using soft lithographic molding. ${ }^{[20,21]}$ PDMS and Ecoflex bond well to each other to form composite structures, are easy to work with, easily accessible, and relatively inexpensive. PDMS has a Shore A hardness of 50, while Ecoflex has a hardness below the Shore A scale (it fractures only above a maximum strain of $900 \%) \cdot{ }^{[22]}$ As a result, PDMS has a limited range of deformation and is well suited for the more rigid part of the tentacle (e.g., the central PneuNet channel, which bends but does not stretch). Ecoflex is more flexible than PDMS and is, therefore, more suitable for the pneumatic channels because they have the largest strain and displacement.

We chose embedded pneumatic networks (PneuNets) as the basis of our strategy for supplying energy to the tentacles primarily because air is light, compressible, readily available, and low in viscosity (and thus permits rapid actuation). The spatial distribution of the pneumatic channels — which expand like connected bladders upon pressurizationdetermines the nature of the resulting actuation. ${ }^{[23]}$ For the tentacles we describe here, the pneumatic networks are based on three indistinguishable channels along the longitudinal direction of the tentacle. Combined actuation of these channels generates movement in three dimensions.

Figure 1 sketches the method we used to fabricate soft tentacles. We designed the masters to mold the elastomers using computer-aided design (CAD; Alibre Inc.). A three- 
dimensional (3D) printer (StrataSys Dimension Elite) generated the masters with acrylonitrile butadiene styrene (ABS) plastic from the CAD files. To facilitate the unmolding of the tentacle from the master, we fabricated each channel template as individual pieces that assemble to form the master. The molds did not require any surface treatment to aid subsequent release of Ecoflex; both the ABS and the silicone elastomer have low interfacial free energies, and adhesion between them is weak. We have used the molds over a hundred times with no apparent damage or degradation in performance.

We formed the PneuNets by casting Ecoflex prepolymer in the mold and curing it at $60{ }^{\circ} \mathrm{C}$ for $15 \mathrm{~min}$. After leaving the ensemble to cool to room temperature, we removed the central channel template by hand and filled the gap with PDMS prepolymer (Figure 1E). After curing was complete, we removed the PneuNet templates from the tentacle, and the tentacle from the container, by gently pulling by hand. After unmolding, the resulting elastomeric tentacle has three individual pneumatic channels parallel to a central PDMS channel (see Figure 1F). The symmetry along the long axis of the structure assures the uniform actuation of the tentacle upon pressurization. We filled 5-mm-length sections at both ends of the tentacle with Ecoflex prepolymer; this polymer, after curing at $60{ }^{\circ} \mathrm{C}$ for $15 \mathrm{~min}$, seals the pneumatic channels. To enable pneumatic activation, we delivered compressed air (25-300 mbar) through polyethylene tubing inserted into the PneuNets (see Figures S4, S5 in the supporting information for details). The total time required to fabricate a tentacle, once the molds have been fabricated, is approximately 3 hours.

Figure 2 shows a tentacle with three individual channels. The total length of the tentacle is $15 \mathrm{~cm}$. The lengths of the pneumatic channels are $14 \mathrm{~cm}$, starting $5 \mathrm{~mm}$ from the end of the tentacle. When pressurized, the pneumatic channels of the tentacle expand in the regions that are most compliant, or that have the lowest stiffness (the regions fabricated in Ecoflex). The expansion of the pneumatic channel thins the Ecoflex walls not in contact with 
the PDMS central channel. To accommodate the asymmetric elongation of the two opposite walls of the channel, the structure surrounding the expanding volume bends around the axis of the channel. When the pressure is sufficiently high ( $230 \mathrm{mbar})$ the Ecoflex in the vicinity of the pneumatic channels goes through a snap through intability ${ }^{[24]}$ (a metastable regime in the stress-strain curve in which large changes in strain require essentially no additional stress); in this regime, parts of the tentacle bend substantially with only a small increase in pressure. At the beginning of the actuation, the bending concentrates at the free end of the tentacle, where the force to initiate the deformation (and the snap-through instability) is less than in the center. This structure-selective regional actuation is one of the many useful non-linearities that make these systems attractive as structures with which to generate complex motions using simple controls. Once the end of the tentacle reaches the snap-through instability, the deformation at the end of the pneumatic channel saturates; the center of the bending motion then propagates towards the root of the tentacle, bending the tentacle in a circular pattern (multiple times, if it is sufficiently long). Since actuation of multiple channels has a synergistic effect on bending; coupled pressurization of several channels can thus bend the tentacle along any axis, and generate three-dimensional motion.

The analytical description of expanding thin-walled balloons is well developed, ${ }^{[25,26]}$ but the complex composite structure used here does not have a simple analytical model. We can, however, use finite-element analysis to model the expanding pneumatic channels of the tentacle (see Experimental section). Figure 2B shows the structural deformation and stress distribution of the cross-section of a micropneumatic channel upon atmospheric pressure and increments of pressure of 75 mbar, 149 mbar, and 231 mbar. Figure 2C shows the curling motion of the tentacle upon pressurization (Figure $\mathbf{S} 7$ shows the simulation of the initiation and propagation of the snap-through instability). 
Tentacles having only one section, with three pneumatic channels distributed along the length of the actuator (Figure 2), can have a single bending mode. To increase the range of motions, and the capability for complex manipulation of the tentacles, we added multiple sections (two to four) to the tentacles, each having three individual pneumatic channels. We embedded the tubes that bring compressed air to each pneumatic channel in the central PDMS channel (a region that bends but does not expand); this design reduced limitations to the mobility of the tentacle (Figure 3A, B). The pressurization of channels from different sections allows multiple bending modes (Figure 3C). Tentacles with multiple sections can adopt complex shapes (Figure 3E-F) and manipulate delicate objects (Figure 3D). In these experiments (Figures 3E-D), we initially placed the objects close to the tentacle so it could reach them by the sequential inflation of its sections. In practical applications, these structures (as grippers) would be part of other structures that provide a greater range of mobility, but less dexterity.

Adding additional functional components to the tentacles improves their capabilities. Figure 4 shows three examples of tentacles that have flexible components (such as wires or tubing) embedded in the central channel; these components do not affect the mobility of the tentacle, but they do add function to it.

Figure 4A shows a tentacle with a video camera implanted at its end. The wires to power the camera and transfer the video signal were inserted along the central channel and glued in place with PDMS. To avoid damage to the video camera from the thermal curing step, the PDMS was cured at room temperature for 48 hours. This tentacle has two sections: the section of the tentacle closest to the tether bends to form a base so the section with the video camera can be lifted out of plane and oriented in a particular direction.

Figure 4B shows a tentacle that has additional tubing embedded along its central channel. This tubing ends in a hypodermic needle $\left(22 \mathrm{G} 1^{1 / 2}\right)$ so fluid can be delivered or 
pumped out of the end of the tentacle. Figure 4C (Supporting Information Figure S9 describes the full sequence) shows a similar tentacle (designed to deliver organic solvents through the central channel); we used this tentacle to deactivate a circuit enclosed in a foamed polystyrene container by delivering, in sequence, acetone (to dissolve the polystyrene) and nitric acid (to dissolve wires in the circuit).

Figure 4D shows a tentacle with a suction cup made of Ecoflex attached to its end. This tentacle has an extra tube embedded in the PDMS central channel; this tube can apply reduced pressure. We directed this tentacle to attach the suction cup to the top of a bottle (see Figure 4E), and then lifted the bottle (Supporting Information Figure $\mathbf{S} 10$ describes the full sequence).

We were also able to use the suction channel to transport solids, for example, granular media ( $0.25 \mathrm{~mm}$ diameter colored glass beads; see Supporting Information video). The transport of particles with diameter over $1 \mathrm{~mm}$ through the central channel is, however, difficult due to clogging. In order to overcome that difficulty, and to be able to transport larger samples of solids, we first dissolved them (here, in hot water).

Figure 5 shows a tentacle with two channels for fluid delivery (FD) and one central channel for suction. By delivering hot water $\left(60{ }^{\circ} \mathrm{C}\right)$ through the $\mathrm{FD}$ tubes onto $\mathrm{NaCl}$ crystals (1-3 mm diameter), we dissolved the salt. After dissolution, we used reduced pressure to transport the salt water through the suction channel (Figure 5E). The water delivered by the tentacle was colored with blue ink to facilitate the visualization of the solution during the mass transport process. 
Tentacles interact with objects by contacting them with their elastomeric skins. The molding technique we use to fabricate tentacles allows the fabrication of tentacles with tailored surfaces, by modifying the molds (Figure S2). We improved the abilities of the tentacles to manipulate smooth or slippery objects by modifying their contacting surfaces. For example, we have fabricated a tentacle with a textured surface that is able to manipulate slippery objects. Figure 6 shows a tentacle with a textured surface that is both more compliant than a flat surface of the same material, and also provides more traction. ${ }^{[27]}$ To demonstrate the improvement in the ability of the tentacle to grip objects with slippery surfaces, we picked up a flat wrench ( $30 \mathrm{~g}$ ) coated with gelatin (to make it more slippery) using a tentacle with a textured surface (Figure 6C-D); this task is one in which similar tentacles with non-textured surfaces failed (an untextured tentacle would pick up the wrench without the gelating coating). Other physical or chemical modifications to the surface of the tentacle could also be applied. ${ }^{[28]}$

This research demonstrates a new method for the fabrication of pneumatically actuated tentacles based on composite elastomeric structures. To move in three dimensions, these tentacles take advantage of the asymmetric deformation of the embedded pneumatic channels upon pressurization. Multiple bending modes can easily be accessed by tentacles with independent micropneumatic sections. The ability of these tentacles to hold, pick up, and manipulate complex shapes and delicate objects demonstrates their versatility.

The functionality of the tentacles increases when components with optoelectronic or mechanical functionality are embedded in them. The tethers for these functional components (wires or tubes) can be embedded in their central channel. Examples include a video camera, a needle with a delivery channel, a suction cup connected to a suction channel. The combination of delivery and suction channels allows the tentacle to transport solids after their 
dissolution. Modifying the topography of the surface of the tentacle improves its ability to hold soft or slippery objects.

When more highly developed, these pneumatic 3D tentacles have the potential to be useful as soft actuators that can manipulate soft and fragile objects, to operate in confined and hazardous spaces, and to perform complex motions. The structure and logic of the controller that will serve best to operate these structures remains to be determined.

The method we use to fabricate soft pneumatic tentacles is simple, fast, and relatively inexpensive. This process is also compatible with techniques such as plastic modeling and extrusion. The tentacles described here have four characteristics that make them especially suited for soft robotic applications: i) They are light (with a mass of $100 \mathrm{~g}$ per meter of tentacle). ii) They are compatible with high-speed actuation (complete activation in $\sim 0.5 \mathrm{~s}$ using air at 300 mbar above atmospheric pressure, delivered through a $25-\mathrm{cm}$ long tether with an internal diameter of $1.57 \mathrm{~mm}$ ). iii) They are resistant to damage from impact and fall (the full function of the tentacle illustrated in Figure 2 remained intact after a fall from heights over 10 meters). iv) They are compatible with the introduction of components with specialized optical, electrical, or mechanical functions, and with the modification of their surface to improve their interaction with objects.

The fabrication method described here, at its present level of development, also has three limitations: i) The tether embedded in the tentacles with more than three pneumatic channels to distribute the gas makes the miniaturization of complex tentacles below the $\mathrm{cm}$ scale difficult. ii) The materials we now use to fabricate the tentacles, and the pneumatic pressures we employ to inflate them, are not suitable for manipulating heavy ( $>200 \mathrm{~g}$ ) objects. The fabrication method, however, is versatile and generalizable and, with an appropriate choice of materials, could be extended to applications that require high loads. The activation of the tentacles fabricated in materials with higher Young's moduli, using fluids 
rather than gas (e.g., hydraulic actuation) is compatible with similar designs and will provide higher force than pneumatic actuation, albeit typically with slower motions. iii) The soft surface of these pneumatic tentacles makes them susceptible to damage when manipulating sharp and pointy objects. We describe the use of alternative composite materials to avoid bursting of the pneumatic channels in subsequent papers, as well as alternatives to tethering, in other papers. ${ }^{[23,29]}$

Experimental

Design of $3 D$ CAD Models: We used software for solid modeling from Alibre Inc. (http://www.alibre.com) for the molds designed in this work.

Fabrication of Molds for Elastomer Casting: A 3D printer (StrataSys, Dimension Elite) using acrylonitrile butadiene styrene (ABS) plastic generated the masters to mold the elastomers from the CAD design. Figure $\mathbf{S} \mathbf{1}$ shows the different pieces that comprise the mold used. The 3D printer required 3 hours to build this mold. After casting and curing the elastomers, we disassembled the mold one piece at a time by hand, pulling carefully to avoid damage to the tentacles or the different parts of the mold. ABS molds can be used multiple times, since the elastomers used to fabricate the tentacles readily release from them.

Preparation of Ecoflex: Ecoflex 00-30 was obtained from Smooth-On (http://www.smooth-on.com). We mixed Ecoflex 00-30 precursors in a 1:1 ratio by volume and degassed the prepolymer, to remove air bubbles, in a desiccator at 36 Torr for $5 \mathrm{~min}$. Curing times ranged from 15 to 30 minutes at $60{ }^{\circ} \mathrm{C}$. 
Preparation of PDMS: Polydimethylsiloxane (PDMS, Sylgard 184) was purchased from Dow Corning. We mixed the precursors of the base to a cross-linking agent at the ratio of 10:1 by weight. The prepolymer mixture was degassed at 36 Torr for 30 min to remove any air bubbles in the mixture, and to insure complete mixing between the two parts. To cure PDMS, we baked the prepolymer mixture at $60^{\circ} \mathrm{C}$ for 2 hours; this curing time and temperature assures a good bond between PDMS and Ecoflex when PDMS is cured in contact with Ecoflex.

Pneumatic Activation: The compressed air supplied for actuation of the PneuNets came from plastic syringes or other sources of compressed air. The air pressure was delivered through a $25-\mathrm{cm}$ long polyethylene tube with an internal diameter of $1.57 \mathrm{~mm}$.

Finite Analysis Simulations: To simulate the pneumatic actuation of the tentacles we used the finite element software ABAQUS to build up a 3D model. Pressure added in one of the pneumatic channels loaded the system initially. We used the Riks method in the simulation to capture the details of the snap-through instability [24]. PDMS was modeled as an incompressible Neo-Hookean material with shear modulus $\mathrm{G}=1.84 \mathrm{MPa}$. In order to capture the snap-through instability, Ecoflex must be modeled as a rubber material that stiffens at high strain. The Supporting Information (Figure S6) shows the experimental relationship between stress and strain for Ecoflex under uniaxial tension. We fitted the data, and modeled Ecoflex, as an incompressible Arruda-Boyce material with shear modulus $\mathrm{G}=0.03 \mathrm{MPa}$ and $\lambda_{\lim }=3.9$. 
Acknowledgements

DARPA supported this work under contracts W911NF-11-1-0094 and

W911NF-09-1-0476. Fabrication and testing of tentacles with multiple sections for complex manipulation was supported by the Department of Energy (DE-FG02-00ER45852). R.V.M.

acknowledges funding by the FP7 People program under the project Marie Curie

IOF-275148. J.L.B. acknowledges the 2011 Harvard REU program (NSF DMR-1005022) and the Wyss Institute for Biologically Inspired Engineering. C.R.F. acknowledges the 2010

Harvard REU program (NSF PHY-0646094) and MRSEC (NSF DMR-0820484). Supporting Information is available online from Wiley InterScience or from the author.

Received: ((will be filled in by the editorial staff))

Revised: ((will be filled in by the editorial staff)) Published online: ((will be filled in by the editorial staff)) 
[1] S. Vogel, Comparative Biomechanics: Life's Physical World, Princeton Univ. Pr., Princeton, USA, 2003, Ch. 3.

[2] D. Trivedi, C. Rahn, W. Kier, I. Walker, Appl. Bionics Biomech. 2008, 5, 99.

[3] G. Sumbre, G. Fiorito, T. Flash, B. Hochner, Nature 2005, 433, 595.

[4] R. Cieslak, A. Morecki, Robotica 1999, 17, 11.

[5] M. W. Hannan, I. D. Walker, Adv. Robotics 2001, 15, 847.

[6] F. Ilievski, A. D. Mazzeo, R. F. Shepherd, X. Chen, G. M. Whitesides, Angew. Chem.

2011, 123, 1930 .

[7] R. F. Shepherd, F. Ilievski, W. Choi, S. A. Morin, A. A. Stokes, A. D. Mazzeo, X.

Chen, M. Wang, G. M. Whitesides, Proc. Natl. Acad. Sci. U.S.A. 2011, 108, 20400.

[8] K. Suzumori, A. Koga, H. Riyoko, Proc. IEEE MEMS 1994, 136.

[9] S. Konishi, M. Nokata, O. Jeong, T. Sakakibara, S. Kusuda, M. Kuwayama, H.

Tsutumi, ISR 2005, 9, 54.

[10] R. Buckingham, A. Graham, Ind. Robot 2005, 32, 120.

[11] D. Trivedi, D. Dienno, C. Rahn, J. Mech. Design 2008, 130, 091402.

[12] X. L. Wang, I. K. Oh, J. Lu, J. Ju, S. Lee, Mater. Lett. 2007, 61, 5117.

[13] Y. Liu, H. Lv, X. Lan, J. Leng, S. Du, Compos. Sci. Technol. 2009, 69, 2064.

[14] Y. Bar-Cohen, J. Adv. Mater. 2006, 38, 3.

[15] G. Kovacs, P. Lochmatter, M. Wissler, Smart Mater. Struct. 2007, 16, S306.

[16] Y. Bar-Cohen, Q. Zhang, MRS Bull. 2008, 33, 173.

[17] D. Trivedi, A. Lotfi, C. D. Rahn, IEEE T. Robotic. Autom. 2008, 24, 773. 
[18] D. Braganza, D. M. Dawson, I. D. Walker, N. Nath, IEEE T. Robotic. Autom. 2007, 23, 1270.

[19] M. B. Pritts, C. D. Rahn, IEEE Int. Conf. Robotic. Autom. 2004, 4742.

[20] D. Qin, Y. Xia, G. Whitesides, Nat. Protoc. 2010, 5, 491.

[21] Soft Lithography for Dummies:

http://www.ifm.liu.se/applphys/biorgel/education/mikrosystem-nanobiologi-t/material-samttipstricks/Soft_Lithography_for_Dummies.pdf (accessed July 2012).

[22] According to the manufacturer's data sheet:

Ecoflex: http://www.smooth-on.com/index.php?cPath=1130 (accessed July 2012).

Paper: https://www.vwrsp.com/catalog/product/index.cgi?catalog_number=21913-211 (accessed July 2012).

[23] R. V. Martinez, C. R. Fish, X. Chen, Adv. Funct. Mater. 2012, 22, 1376.

[24] R. Seydel, Practical Bifurcation and Stability Analysis, Vol. 5, Springer, New York, USA, 2010, Ch. 4 .

[25] E. Chater, J. Hutchinson, J. Appl. Mech. 1984, 51, 269.

[26] A. Gent, Rubber Chem. Technol. 1999, 72, 263.

[27] A. Ghatak, L. Mahadevan, J. Y. Chung, M. K. Chaudhury, V. Shenoy, P. Roy. Soc. Lond. A Mat. 2004, 460, 2725.

[28] A. V. Pocius, D. A. Dillard, M. K. Chaudhury, Adhesion Science and Engineering, Elsevier, Dordrecht, Netherlands, 2002, Ch. 6.

[29] C. D. Onal, X. Chen, G. M. Whitesides, D. Rus, ISRR 2011. 


\section{Table of Contents}

Soft robotic tentacles that move in three-dimensions upon pressurization are fabricated by composing flexible elastomers with different tensile strength using soft lithographic molding. These actuators are able to grip complex shapes and manipulate delicate objects. Embedding functional components into these actuators (for example, a needle for delivering fluid, a video camera, and a suction cup) extends their capabilities.

Keywords: Soft Robotics, Tentacles, Pneumatic Actuators, Three-dimensional Motion, Composites.

R. V. Martinez, J. L. Branch, C. R. Fish, L. Jin, R. F. Shepherd, R. M. D. Nunes, Z. Suo, and G. M. Whitesides $^{*}$

Robotic Tentacles with Three-Dimensional Mobility Based on Flexible Elastomers

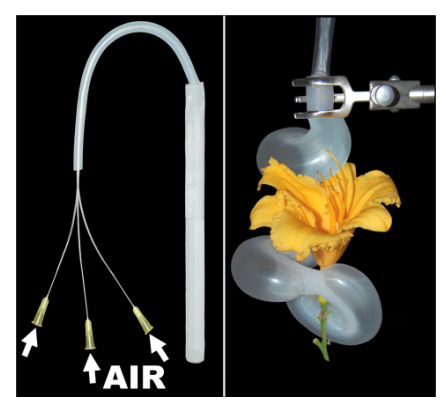




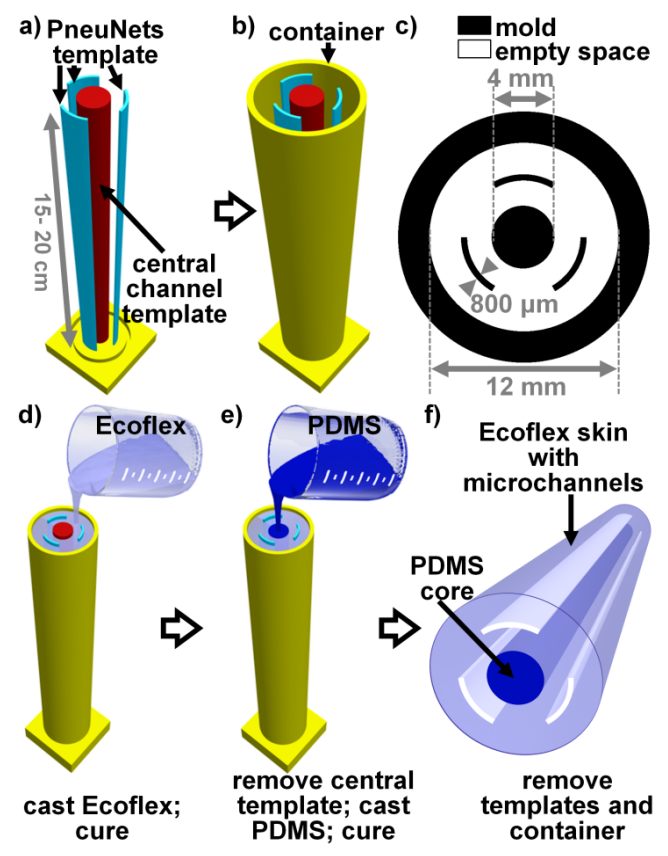

Figure 1. Fabrication of 3D tentacles. a) Mold with the templates for the PneuNet and the central channel templates. b) The mold is placed into a container having the final outer diameter of the tentacle. The channel templates and container are parallel. c) Top view of the mold with the dimensions of the cross section of the tentacle. d) The mold is filled with Ecoflex and cured at $60{ }^{\circ} \mathrm{C}$ for 15 min. e) Removing the central channel allows PDMS to be poured into that space. PDMS and Ecoflex bond together after curing for $2 \mathrm{~h}$ at $60{ }^{\circ} \mathrm{C}$. f) The tentacle is removed from the mold. 


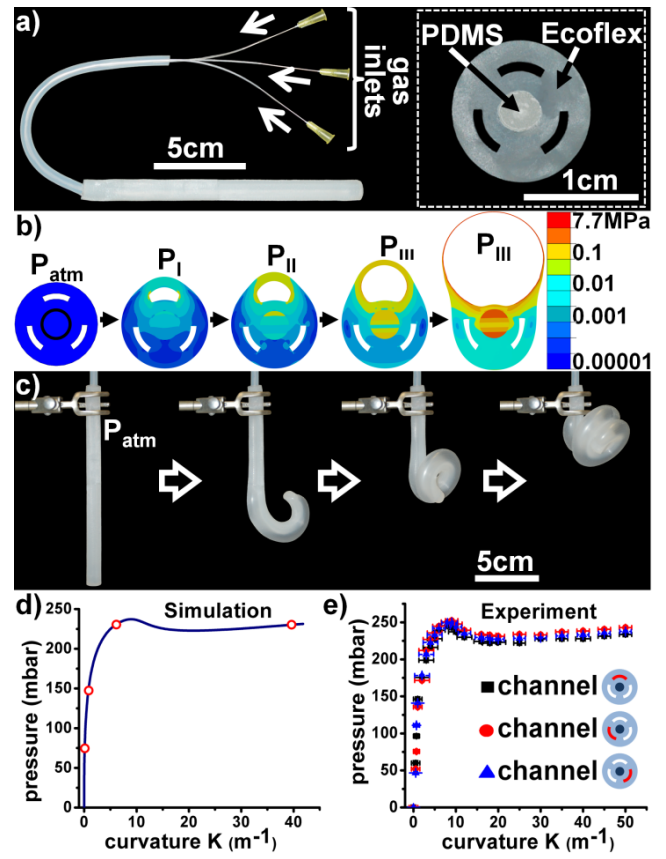

Figure 2. Movement of a tentacle. a) Tentacle with connectors to the external pressure source. The inset shows the cross section of the tentacle. b) Finite-element analysis describing the expansion of the micropneumatic channel upon progressive pressurization from atmospheric pressure $\left(\mathrm{P}_{\mathrm{atm}}\right), \mathrm{P}_{\mathrm{I}}=75 \mathrm{mbar}, \mathrm{P}_{\mathrm{II}}=149 \mathrm{mbar}, \mathrm{P}_{\mathrm{III}}=231 \mathrm{mbar}$ (before and after the snap-through instability). These values for the pressure are shown as dots in d). The color bar indicates the von Mises stress distribution. c) Progressive bending of the 3D tentacle by pressurization of one of its channels. d) Simulation of the curvature of the tentacle with pressure. e) Experimental dependence on pressure of the curvature of each channel after 50 pressurization/depressurization cycles. 

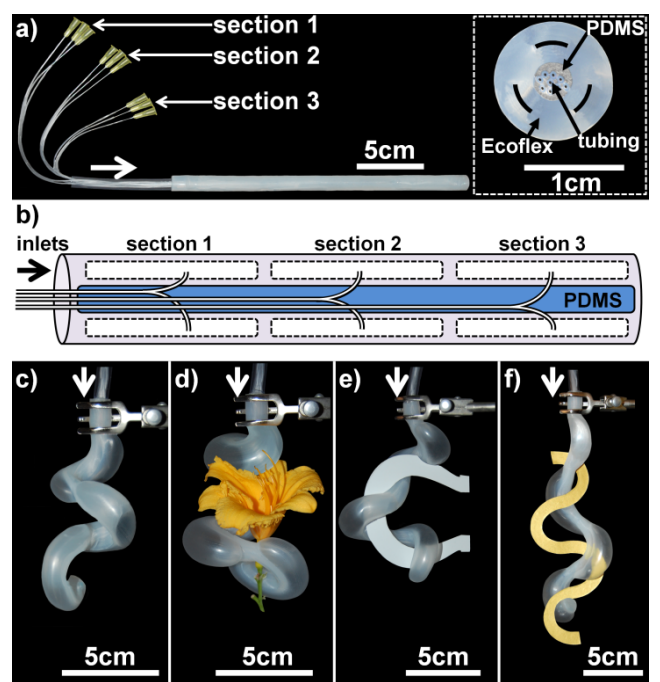

Figure 3. 3D tentacles with multiple sections. a) Three-sectioned tentacle with nine independent microchannels, each controlled independently by an external source of pressure. The inset shows the cross section of the tentacle with the tubing traveling along the PDMS central channel. b) Schematic describing the distribution of the tubing inside the tentacle (not to scale). c) Tentacle with three sections inflated simultaneously. d) Tentacle holding a flower by conforming its three sections to the flower. e) Tentacle holding a horseshoe-shaped object. f) Tentacle with four sections holding a complex shape. 


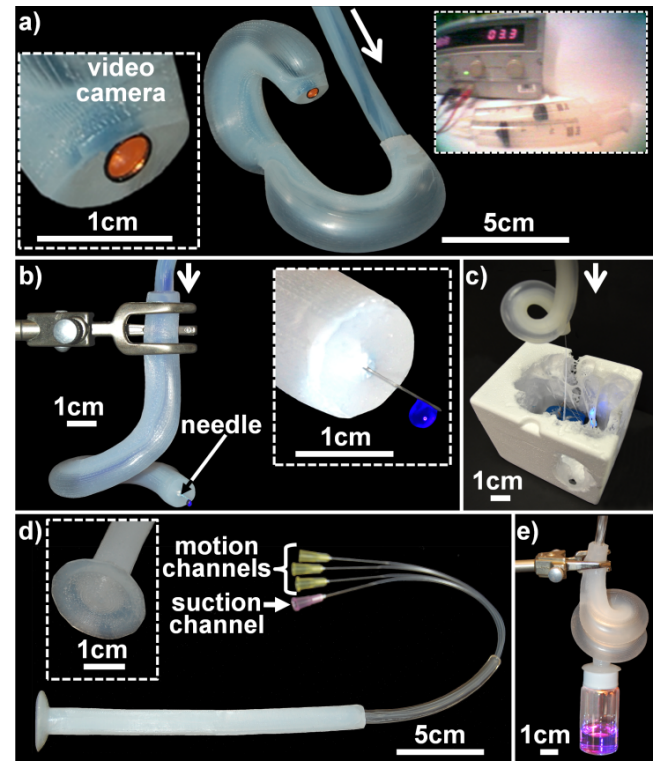

Figure 4. Images of different tentacles with embedded functionality. a) Tentacle with two sections; this structure incorporates a fully operational video camera on its end. Inset on the left shows a close-up image of the end of the tentacle, where the embedded camera protrudes. Inset on the right is a picture taken from this camera showing the two syringes used for the pneumatic actuation and the $3.3 \mathrm{~V}$ voltage source that powers the camera. b) Tentacle that can transfer liquids through its length by connecting a needle to the additional channel for delivery. c) Soft tentacle dissolving a polystyrene box by directing a stream of acetone over its top. d) Tentacle with a suction cup and a suction channel. e) Tentacle lifting a glass bottle by applying vacuum through a suction cup. 


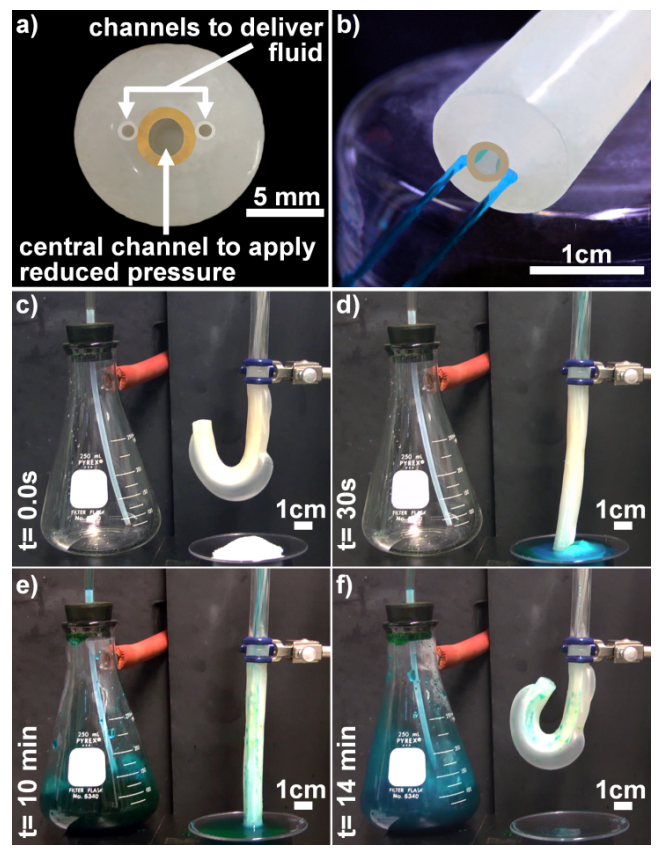

Figure 5. Transport of solids through tentacles. a) Top view of a tentacle with a central silicone tube for applying reduced pressure and two polyethylene tubes to deliver fluids. b) Tentacle delivering colored water through the delivery channels. c-f) Soft tentacle dissolving crystals of $\mathrm{NaCl}(\sim 1-3 \mathrm{~mm}$ size $)$ with $60{ }^{\circ} \mathrm{C}$ colored water. After waiting for the water to dissolve the $\mathrm{NaCl}$, the solution is pumped out to an Erlenmeyer flask through the central channel by applying reduced pressure. 


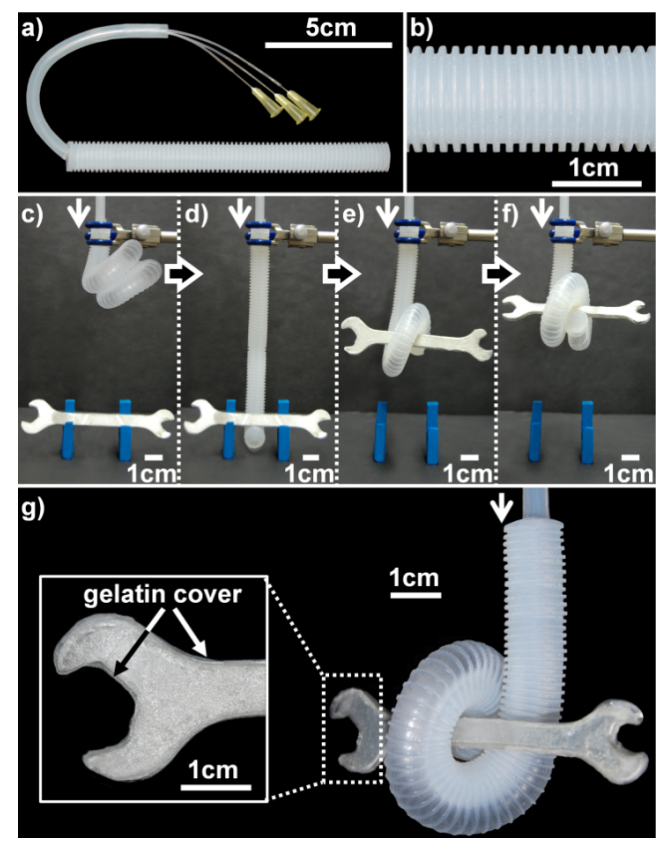

Figure 6. a) Textured tentacle with three inlets. b) Expanded view of the surface of the tentacle showing ridges, which improve traction. c-g) Tentacle holding a metallic wrench coated with gelatin. The textured surface of this tentacle is more compliant than a flat surface because half of the volume has effectively been removed, and each ridge deforms easily. 